\title{
Delicaflavone Reverses Cisplatin Resistance via Endoplasmic Reticulum Stress Signaling Pathway in Non-Small Cell Lung Cancer Cells
}

This article was published in the following Dove Press journal: OncoTargets and Therapy

\author{
Xuewen Wang ${ }^{1, *}$ \\ Bing Chen (D) ${ }^{1-3, *}$ \\ Danfen $\mathrm{Xu}^{\prime}$ \\ Zhijun Li (D) ${ }^{4}$ \\ Yuxia Sui ${ }^{5,6}$ \\ Xinhua $\operatorname{Lin}^{1-3}$
}

'Department of Pharmaceutical Analysis, School of Pharmacy, Fujian Medical University, Fuzhou, Fujian, People's Republic of China; ${ }^{2}$ Nano Medical Technology Research Institute, Fujian Medical University, Fuzhou, Fujian, People's Republic of China; ${ }^{3}$ Higher Educational Key Laboratory for Nano Biomedical Technology of Fujian Province, Fujian Medical University, Fuzhou, Fujian, People's Republic of China; ${ }^{4}$ School of Pharmacy, Fujian Medical University, Fuzhou 350180, Fujian, People's Republic of China; ${ }^{5}$ Department of Pharmacy, Shengli Clinical Medical College of Fujian Medical University, Fuzhou 35000I, People's Republic of China; ${ }^{6}$ Department of Pharmacy, Fujian Provincial Hospital, Fuzhou 35000I, People's Republic of China

*These authors contributed equally to this work

Correspondence: Xinhua Lin Department of Pharmaceutical Analysis, Fujian Medical University, No. I Xueyuan Road, University Town, Fuzhou 350I22,

People's Republic of China

$\mathrm{Tel} / \mathrm{Fax}+86$ I3906909638

Email xhlfz|2345432।@sina.com

Yuxia Sui

Department of Pharmacy, Fujian

Provincial Hospital, No. I 34 Dong Street,

Fuzhou 35000I, People's Republic of

China

Tel/Fax +86 I5959090652

Email suiyuxia123@I63.com
Background: The incidence and mortality of lung cancer continue to increase around the world; in 2018, new lung cancer cases accounted for $11.6 \%$ of all cancer cases, and lung cancer deaths accounted for $18.4 \%$ of cancer deaths. Cisplatin (DDP) is a first-line chemotherapy drug for lung cancer; however, DDP resistance can lead to a poor prognosis in patients with lung cancer. Therefore, reversing DDP resistance is a treatment goal.

Materials and Methods: Cell counting kit-8 (CCK8) assays, wound healing analyses, Transwell assays, in vitro tumor xenografts, and flow cytometry were used to detect the proliferation, migration, invasion, and apoptosis of multidrug resistant A549/DDP and PC9/ DDP cells, respectively. Western blot was performed to detect protein levels of cleaved caspase-3, CHOP, and GRP78.

Results: Delicaflavone inhibited DDP resistance of lung cancer cells and decreased proliferation in a dose- and time-dependent manner. It also decreased migration and invasion and enhanced apoptosis. Western blots showed that delicaflavone overcame DDP resistance by increasing the expression of GRP78 and CHOP and the apoptosis-related protein cleaved caspase-3.

Conclusion: Delicaflavone can reverse DDP resistance in A549/DDP and PC9/DDP cells by inhibiting cell proliferation and migration and enhancing apoptosis and cleaved caspase-3 levels by increasing the expression of CHOP and GRP78 protein via the endoplasmic reticular stress pathway. It could be a useful therapeutic adjunct to treat DDP-resistant lung cancer.

Keywords: delicaflavone, Cisplatin; DDP, lung cancer, endoplasmic reticulum stress

\section{Introduction}

Lung cancer incidence and mortality continue to increase worldwide; in 2018, new lung cancer cases accounted for $11.6 \%$ of all cancer cases, and lung cancer deaths accounted for $18.4 \%$ of all cancer deaths. ${ }^{1-3}$ Because early clinical manifestations of lung cancer are not typical, early detection and treatment are limited. Chemotherapy is the primary treatment for middle- and late-stage lung cancer. ${ }^{4,5}$ Cisplatin (DDP) is a chemotherapeutic drug widely used in the treatment of lung cancer, but drug resistance of tumors had affected its application. ${ }^{6}$ Exploring ways to reverse DDP drug resistance will improve the treatment of lung cancer.

Delicaflavone is a biflavonoid compound existing in the Selaginella doederleinii Hieron and other herbals or plants. It has good antitumor activity and excellent safety profiles. It is worth further research and development. Previous studies 
showed that Delicaflavone could induced apoptosis, autophagy, cell cycle arrest and inhibits PI3K/AKT/mTOR, Ras/ MEK/Erk, Akt/mTOR/p70S6K signaling pathways and MAPK signaling cascades in colorectal cancer cell, lung cancer and cervical cancer HeLa cells. ${ }^{7-9}$ However, it has not been reported whether delicaflavone can reverse DDP resistance in the human lung adenocarcinoma multidrug resistant A549/DDP and PC9/DDP cell line. The aims of this study is to investigate DDP resistance reversal by delicaflavone in A549/DDP and PC9/DDP cells and explore its possible molecular mechanism.

Our results confirmed that delicaflavone could attenuate DDP resistance in A549/DDP and PC9/DDP cells by inhibiting cell proliferation and migration and enhancing apoptosis and cleaved caspase- 3 levels by increasing the expression of CHOP and GRP78 protein via the endoplasmic reticular stress pathway. It could be a useful therapeutic adjunct to treat DDP-resistant lung cancer.

\section{Materials and Methods Cell Culture}

A549, A549/DDP, PC9, and PC9/DDP were purchased from the Cell Collection Committee of the Chinese Academy of Sciences (Shanghai, China) and the cells were cultured with RPMll640+10\% FBS at $37^{\circ} \mathrm{C}, 5 \% \mathrm{CO}_{2}$.

\section{CCK8 Analysis}

$5 \times 10^{3} /$ well A549, A549/DDP, PC9, and PC9/DDP cells were inoculated into 96 well plates for $12 \mathrm{~h}$, then, the different concentrations of delicaflavone $(5,10,20,40$ and $80 \mu \mathrm{M})$ or DDP $(2,4,8,16$ and $32 \mu \mathrm{M})$ were added. In the meantime, A549/DDP cells were cultured with delicaflavone $(20 \mu \mathrm{M})$ or DDP $(16 \mu \mathrm{M})$ or delicaflavone $(20 \mu \mathrm{M})+$ DDP $(16 \mu \mathrm{M})$. The control group was added with an equal volume of PBS, and each group was repeated 3 times. $22 \mathrm{~h}$ after, incubation with $10 \mu \mathrm{L}$ CCK- 8 (Sigma-Aldrich, USA) solution for $2 \mathrm{~h}$, The OD value at the wavelength of $450 \mathrm{~nm}$ was measured by microplate assay (EnSpire 2300, PerkinElmer, USA), and the cell growth curve was measured according to the OD value.

\section{Wound Healing}

A549/DDP and PC9/DDP cells density were adjusted to make $2 \times 10^{6} / \mathrm{mL}$ cell suspension absord $0.2 \mathrm{~mL}$ and inoculated into a 6-well plate. Twenty-four hours later, delicaflavone $(20 \mu \mathrm{M})$ or DDP $(16 \mu \mathrm{M})$ or delicaflavone $(20 \mu \mathrm{M})$ + DDP $(16 \mu \mathrm{M})$ were added into culture bottle for $24 \mathrm{~h}$. The control group was added with an equal volume of PBS, and each group was repeated 3 times. We observed the width of the scratch under the inverted microscope and take photos.

\section{Transwell Assay}

A549/DDP, PC9/DDP cells were cultured with Delicaflavone $(20 \mu \mathrm{M})$ or $\operatorname{DDP}(16 \mu \mathrm{M})$ or Delicaflavone $(20 \mu \mathrm{M})+\mathrm{DDP}(16 \mu \mathrm{M})$ for $24 \mathrm{~h}$. The control group was added with an equal volume of PBS, and each group was repeated 3 times. $4 \times 10^{4} /$ well A549/DDP, PC9/DDP cells were inoculated onto the upper chamber with $2 \%$ FBS and $500 \mathrm{~mL}$ medium containing 10\% FBS was added to the lower chamber. Incubated for $24 \mathrm{~h}$, removal of the upper chamber of cells, the cells were fixed with $4 \%$ paraformaldehyde, stain with crystal violet, washed, dried and counted.

\section{In vitro Tumor Xenografts}

A549/DDP, PC9/DDP cells density were adjusted to make $2 \times 10^{7} / \mathrm{mL}$ cell suspension absord $0.2 \mathrm{~mL}$ and injected into the back of mice. 10d later, delicaflavone $(20 \mu \mathrm{M})$ or DDP $(16 \mu \mathrm{M})$ or delicaflavone $(20 \mu \mathrm{M})+\mathrm{DDP}(16 \mu \mathrm{M})$ peritoneal injection twice a week. The control group was added with an equal volume of normal saline. Observed continuously for 3 weeks and the tumor size was measured by scaler every week, and each group was repeated 3 times. The laboratory animals were approved by the medical laboratory animal ethics committee of Fujian Medical University. Instructive notions with respect to caring for laboratory animals (which is released by the Ministry of Science and Technology of the People's Republic of China in September 30th, 2006) were followed for the welfare of the animals.

\section{Flow Cytometry Assay}

A549/DDP, PC9/DDP cells were cultured with delicaflavone $(20 \mu \mathrm{M})$ or DDP $(16 \mu \mathrm{M})$ or delicaflavone $(20 \mu \mathrm{M})$ +DDP $(16 \mu \mathrm{M})$ for $24 \mathrm{~h}$. The control group was added with an equal volume of PBS, and each group was repeated 3 times. A549/DDP, PC9/DDP cells were digested with trypsin without ED-TA and cell suspension was prepared, then $800 \mathrm{r} / \mathrm{min}$ centrifugation for $10 \mathrm{~min}$ at $4^{\circ}$ C. $5 \mu \mathrm{L}$ Annexin V-FITC and $5 \mu \mathrm{L}$ propidium iodide (PI) (Life technologies, USA) were added to mix. The apoptosis rate was detected by flow cytometry (BD, USA).

\section{Western Blot Assay}

Extraction of total protein and BCA quantitative method was used to detect the protein concentration (Thermo Scientific, 
USA). Then the antibody was added to incubate, cleavedcaspase-3, CHOP, GRP78, $\beta$-actin (Cell Signaling Technology, USA), ECL reagent (Amersham Biosciences) chemiluminescence, development and Image $\mathrm{J}$ was used for analysis, and each group was repeated 3 times.

\section{Statistical Analysis}

SPSS 20.0 statistical software was used to analyze test data. The measurement data is expressed as mean \pm standard ( $\ddot{\mathrm{y}} \pm \mathrm{s}), T$-test was used for comparison between the two groups, and ANOVA was used for comparison between multiple groups. $\mathrm{P}<0.05$ was considered statistically significant $\left({ }^{*} \mathrm{P}<0.05\right.$ represents compared with control group. ${ }^{\#} \mathrm{P}<0.05$ represents compared with the Delicaflavone-alone group or DDP-alone group.)

\section{Results}

\section{Delicaflavone United with DDP Regulated the Cell Viability of Lung Cancer DDP Resistant Cells}

In this study, we firstly examined the cell viability of A549, PC9, A549/DDP, and PC9/DDP by CCK8 assay. The result showed that the cell proliferation inhibitory rate of DDP on A549 and PC9 cells was higher than that of A549/DDP and PC9/DDP cells in a dose-dependent manner (Figure 1A and B), the half maximal inhibitory concentration (IC50) of A549 was $16 \mu \mathrm{M}$ and of A549/DDP cells was $48 \mu \mathrm{M}$ by DDP. A549/ DDP exhibited more significant DDP resistance than A549. In order to investigate the cell proliferation inhibitory effect of delicaflavone on A549/DDP and PC9/DDP cells, CCK8 assay showed delicaflavone can inhibited A549/DDP and PC9/DDP cells proliferation in a dose-dependent manner and the nonlethal concentration of A549/DDP and PC9/DDP were $20 \mu \mathrm{M}$ delicaflavone (Figure 1C and D). Further, the results revealed that $20 \mu \mathrm{M}$ delicaflavone combined with $16 \mu \mathrm{M}$ DDP had a high cooperative proliferation inhibition rate (Figure 1E and F). The cell viability of A549/DDP and PC9/DDP cells were detected by CCK 8 assay at the concentrations of delicaflavone $(20 \mu \mathrm{M})$ or DDP $(16 \mu \mathrm{M})$ or delicaflavone $(20 \mu \mathrm{M})+\mathrm{DDP}(16$ $\mu \mathrm{M})$ for $24 \mathrm{~h}, 48 \mathrm{~h}, 72 \mathrm{~h}$. The results indicated that compare with the delicaflavone united with DDP group, the cell viability of A549/DDP and PC9/DDP cells were significantly lower than that of the delicaflavone-alone group or DDP-alone group (Figure $1 \mathrm{G}$ and $\mathrm{H}$ ), revealing that delicaflavone united with DDP can significantly inhibit the cell viability.
Delicaflavone United with DDP Regulated Lung Cancer DDP Resistant Cells' Invasion, Migration

In order to evaluated whether delicaflavone affects the migration and invasion effect of A549/DDP and PC9/DDP cells. Wound healing showed compared with the control group, the cell migration rate of A549/DDP and PC9/DDP cells were decreased in delicaflavone united with DDP group, delicaflavone-alone group and DDP-alone group, compare with the delicaflavone united with DDP group, the migration ability of A549/DDP and PC9/DDP cells were significantly reduced than that of the delicaflavonealone group or DDP-alone group (Figure 2A-D).

Transwell assay showed compared with the control group, the migration number of cells were decreased in delicaflavone united with DDP group, compared with the delicaflavone-alone group or DDP-alone group, the migration number of cell were decreased was significantly reduced in the delicaflavone united with DDP group (Figure 2E-H). In vitro tumor Xenografts, the results of tumor volume was similar reduced in the delicaflavone united with DDP group (Figure 2I-L). Dates revealed that delicaflavone reversed A549/DDP cells invasion, migration in vivo and in vitro experiments.

\section{Delicaflavone United with DDP Regulated Lung Cancer DDP Resistant Cells Apoptosis and the Expression of Apoptosis-Related Proteins}

To assess whether delicaflavone could affect A549/DDP and PC9/DDP cells apoptosis. Flow cytometry assay was used to evaluate A549/DDP and PC9/DDP cells apoptosis by delicaflavone $(20 \mu \mathrm{M})$ or DDP $(16 \mu \mathrm{M})$ or delicaflavone $(20 \mu \mathrm{M})+\mathrm{DDP}(16 \mu \mathrm{M})$ for $24 \mathrm{~h}$. Flow cytometry assay showed compared with the control group, the apoptosis rate of A549/DDP and PC9/DDP cells were increased in delicaflavone united with DDP group, compared with the delicaflavone-alone group or DDP-alone group. The apoptosis rate was increased in delicaflavone united with DDP group, compared with the delicaflavonealone group or DDP-alone group (Figure 3A-D). Subsequently, Western blot revealed that protein expression level of cleaved-caspase-3 was a similar result in A549/DDP and PC9/DDP cells (Figure 3E-H). 

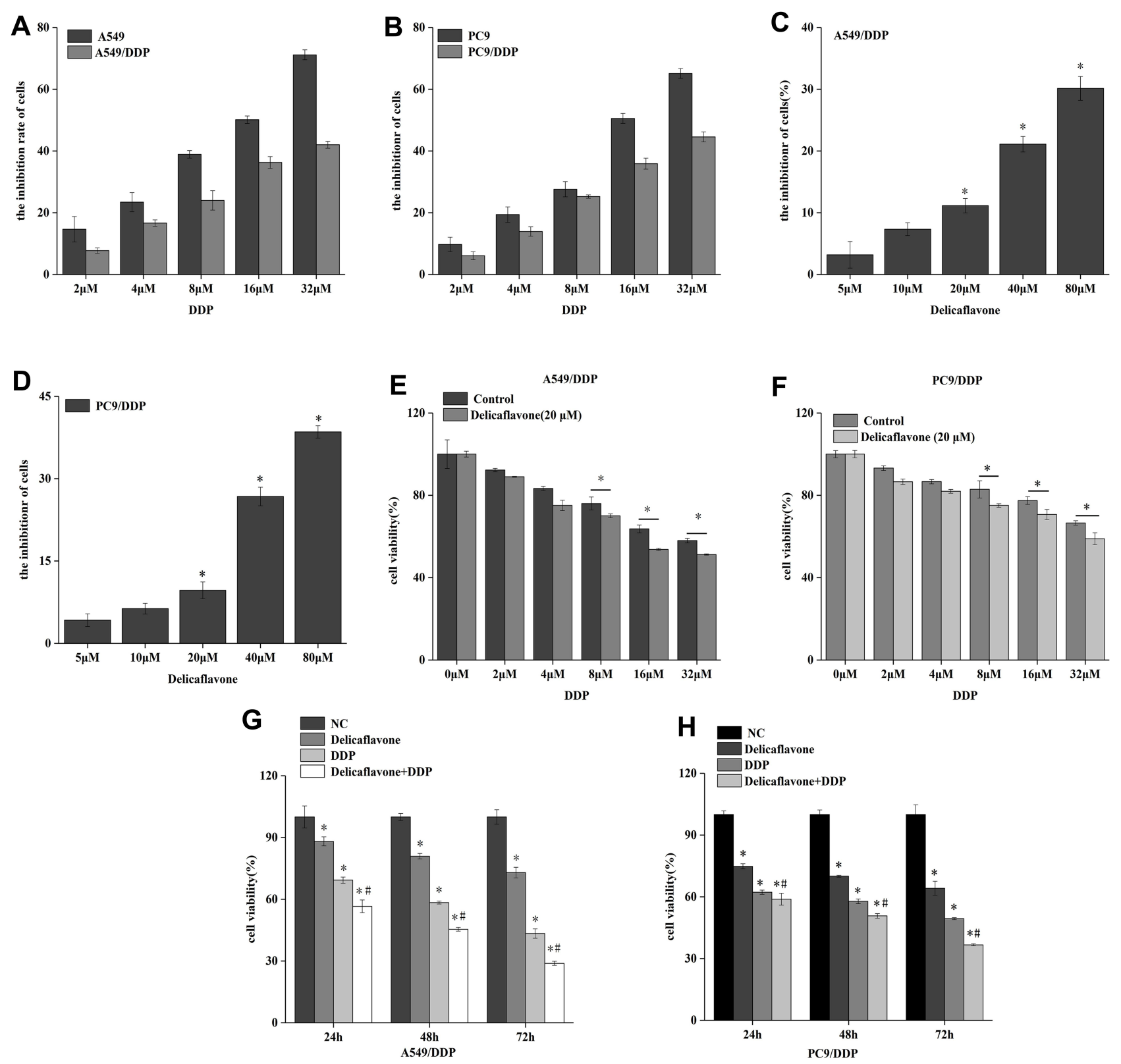

Figure I Delicaflavone united with DDP could reduced the cell viability of lung cancer DDP resistant cells. (A) the cell viability of A549 and A549/DDP cells were detected with the concentrations of DDP for 24h. (B) the cell viability of PC9 and PC9/DDP cells were detected with the concentrations of DDP for 24h. (C) the cell viability of A549 and A549/DDP cells were detected with the concentrations of Delicaflavone for $24 \mathrm{~h}$. (D) the cell viability of PC9 and PC9/DDP cells were detected with the concentrations of Delicaflavone for $24 \mathrm{~h}$. (E) A549/DDP cells were treated with $20 \mu \mathrm{M}$ Delicaflavone combined with indicated concentrations of DDP for $24 \mathrm{~h}$. (F) PC9/DDP cells were treated with $20 \mu \mathrm{M}$ Delicaflavone combined with indicated concentrations of DDP for $24 \mathrm{~h}$. (G) the cell viability of A549/DDP cells were detected by CCK8 at the concentrations of Delicaflavone $(20 \mu \mathrm{M})$ or DDP $(16 \mu \mathrm{M})$ or Delicaflavone $(20 \mu \mathrm{M})+\mathrm{DDP}(16 \mu \mathrm{M})$ for $24 \mathrm{~h}, 48 \mathrm{~h}, 72 \mathrm{~h}$. (H) the cell viability of PC9/DDP cells were detected by CCK8 at the concentrations of Delicaflavone $(20 \mu \mathrm{M})$ or DDP $(16 \mu \mathrm{M})$ or Delicaflavone $(20 \mu \mathrm{M})+\mathrm{DDP}(\mathrm{I} 6 \mu \mathrm{M})$ for $24 \mathrm{~h}, 48 \mathrm{~h}, 72 \mathrm{~h}$. Data represent mean \pm SD. $* \mathrm{P}<0.05$. ${ }^{\#} \mathrm{P}<0.05$ represents compared with the Delicaflavone alone group or DDP alone group.

Delicaflavone United with DDP Regulated Lung Cancer DDP Resistant Cells Invasion, Migration, Apoptosis Through Endoplasmic Reticulum Stress Signaling

\section{Pathway}

To explore whether Delicaflavone affect A549/DDP and PC9/DDP cells invasion, migration, apoptosis by
ER stress signaling pathway. The protein expression of ER stress-related protein CHOP and GRP78 were detected by Western blot. The protein expression of GRP78 and CHOP were increased in DDP group compared with the control group, and also increased in delicaflavone united with DDP group, compared with the Delicaflavone-alone group or DDP-alone group (Figure 4A-D). 

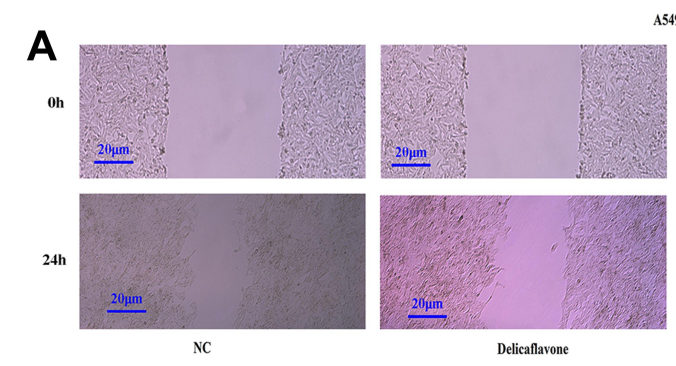

A549/DDP
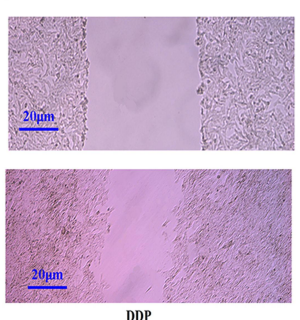

DDP

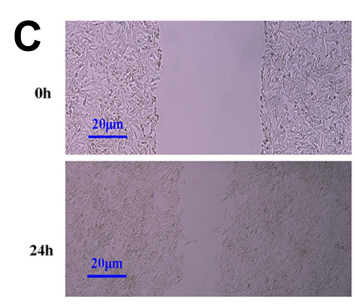

E
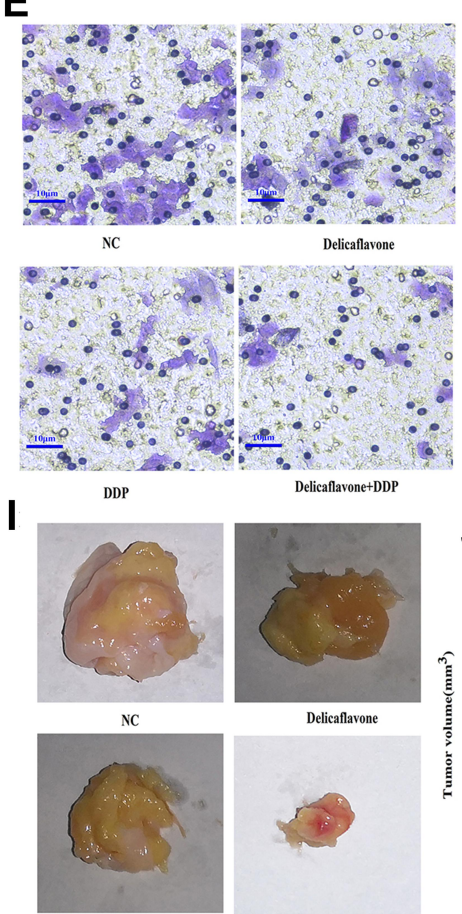

DDP

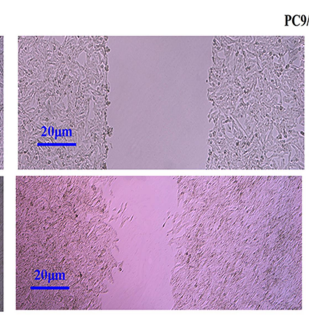

Delicaflavone

F

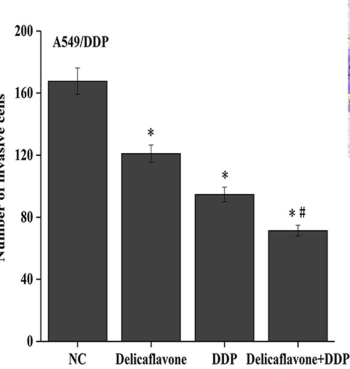

J

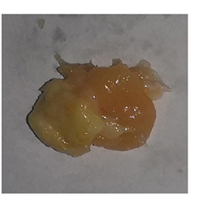

Delicaflavone

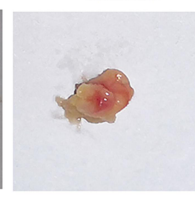

Delicaflavone+DDP

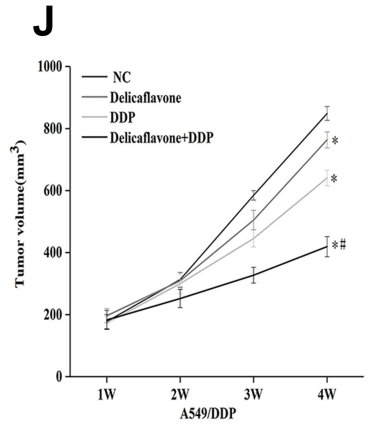

9DDP

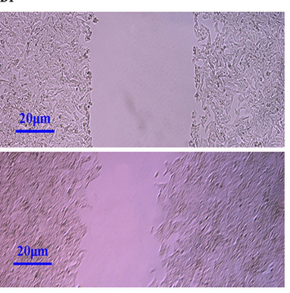

DDP

G

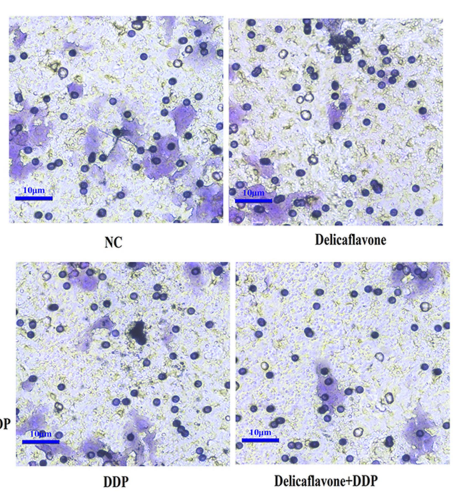

K
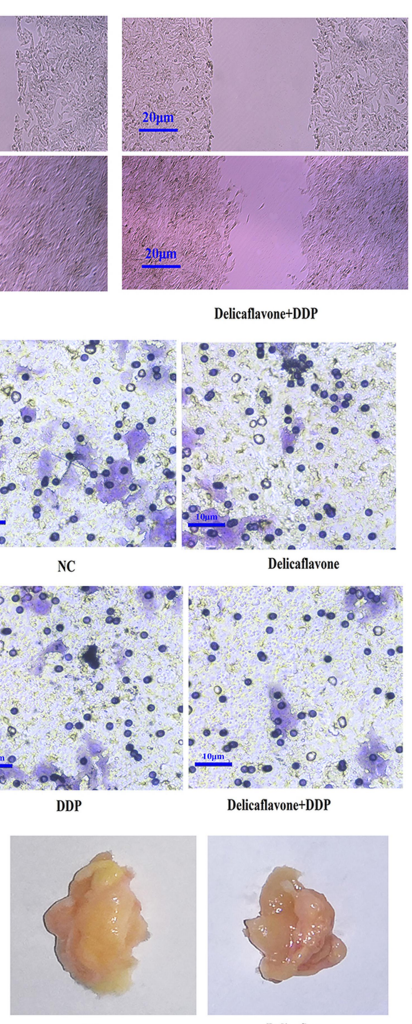

Delicaflavone+DDP

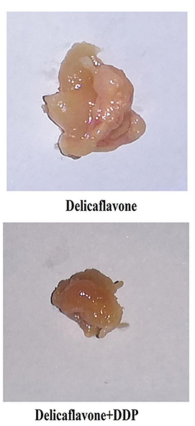

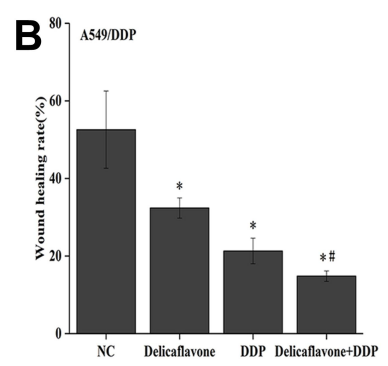

D

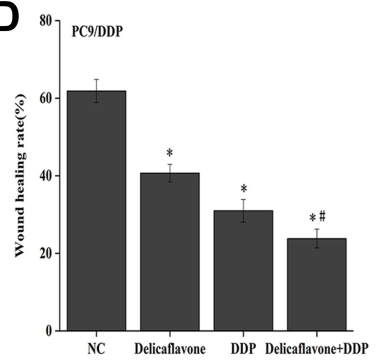

H

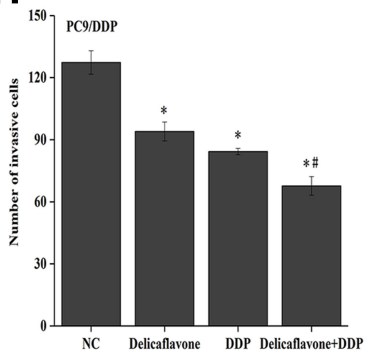

Figure 2 Delicaflavone united with DDP regulated lung cancer DDP resistant cells invasion, migration. (A-D) Wound healing showed the migration ability of the Delicaflavone united with DDP group was significantly reduced than that of the Delicaflavone alone group or DDP alone group. (E-H) Transwell assay showed the migration number of cell were decreased was significantly reduced in the Delicaflavone united with DDP group. (I-L) In vitro tumor Xenografts, the results of tumor volume was reduced in the Delicaflavone united with DDP group. Data represent mean \pm SD. ${ }^{* P}<0.05$. ${ }^{\#} P<0.05$ represents compared with the Delicaflavone alone group or DDP alone group.

\section{Discussion}

Lung cancer has the characteristics of high morbidity and mortality, and non-small cell lung cancer accounts for $85 \%$ of lung cancer. ${ }^{10}$ Chemotherapy is a strategy for the treatment of lung cancer. DDP was a common chemotherapy drug for cancers and plays an anti-tumor role by inducing apoptosis, autophagy and necrosis. ${ }^{11-13}$ However, the drug resistance of DDP in tumor was limited the clinical application. The resistance of lung cancer cells to DDP is a complex process, involving multiple factors and genes, including the mechanisms of the accumulation and deactivation of intracellular drugs, DNA damage repair, autophagy, and apoptosis. ${ }^{14-17}$

Recently more and more research has focused on the mechanism of Delicaflavone in tumors. It exerts antitumor activity by inducing apoptosis and autophagy and 


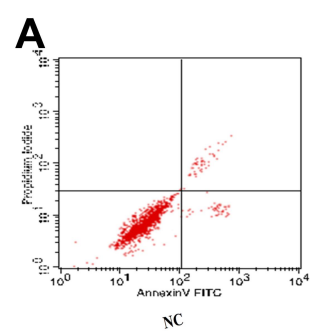

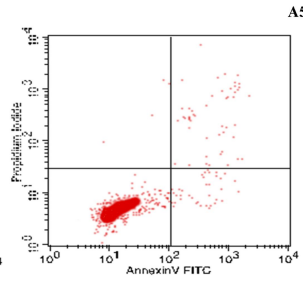

Delicaflavone

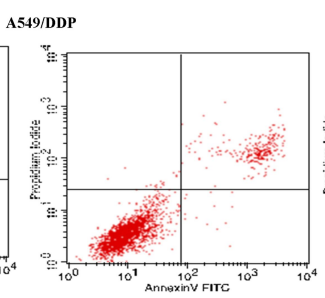

DDP
Delicaflavone $+D D P$
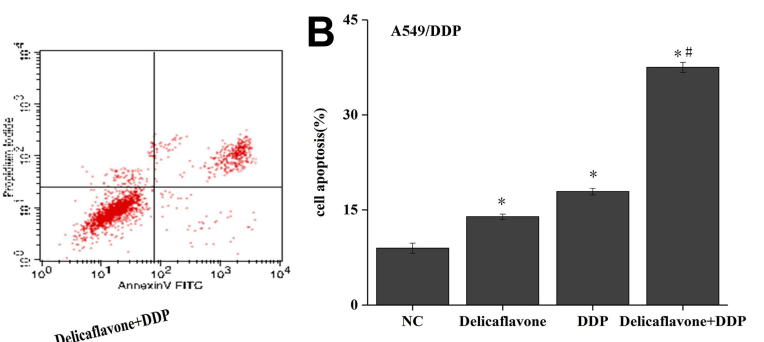

D

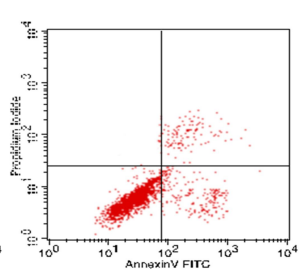

DelicaflavonetDDP
$D_{25}$

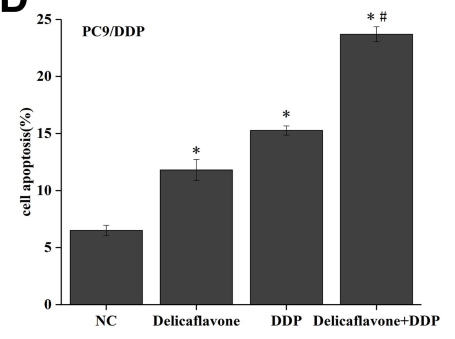

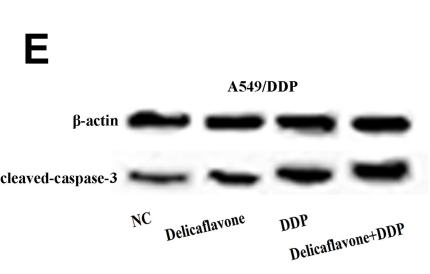

Delicaflavone

AnnoxinV
DDP
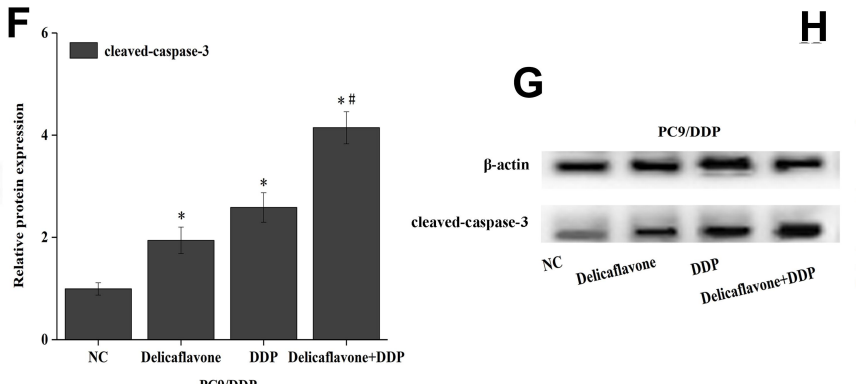

H

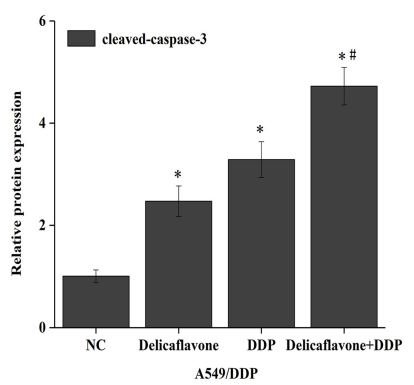

Figure 3 Delicaflavone united with DDP regulated lung cancer DDP resistant cells apoptosis and the expression of apoptosis related proteins. (A-D) Flow cytometry showed that NC, Delicaflavone, DDP, Delicaflavone united with DDP could regulated lung cancer DDP resistant cells apoptosis. (E-H) The protein expression of cleavedcaspase- 3 was detected by Western blot. ${ }^{*} \mathrm{P}<0.05$ represents compared with control group. ${ }^{\#} \mathrm{P}<0.05$ represents compared with the Delicaflavone-alone group or DDPalone group.

inhibiting proliferation in in vitro tumor xenografts of colorectal and cervical cancer cells. ${ }^{7-9}$ However, the research focus on reversing drug resistance was lacked. In this study, Dates revealed that the cell viability of A549/ DDP and PC9/DDP in Delicaflavone united with the DDP group was significantly lower than that of the delicaflavone-alone group or DDP-alone group. Wound healing, Transwell assay, and in vitro tumor xenografts also showed that delicaflavone decreased migration and invasion in vivo and in vitro experiments.

Endoplasmic reticulum (ER) stress is an important cellular protective mechanism. In some tumors, ER stress activates a series of compensatory mechanisms, and discovered that Hypoxia, oxidative stress and drugs can induce ER stress and cleaved-caspase- 3 can promotes apoptosis by promotion the expression of GRP78 and CHOP protein in some tumors. ${ }^{18-21}$ Recent research suggests that ER stress may be related to the molecular mechanism of tumor drug resistance. ${ }^{2-25}$ In our study, Delicaflavone could enhance A549/DDP and PC9/DDP cells apoptosis by Flow cytometry assay, Western blots showed that delicaflavone overcame DDP resistance by increasing the expression of GRP78 and CHOP and the apoptosisrelated protein cleaved caspase-3.

Our results proved that Delicaflavone could attenuate DDP resistance in A549/DDP and PC9/DDP cells by inhibiting cell proliferation, migration, and enhancing apoptosis and cleaved-caspase- 3 protein by increasing the expression of GRP78 and CHOP via the ER stress pathway. This study provided a theoretical foundation for the combination of Delicaflavone and chemotherapy drugs in the treatment of lung cancer.

\section{Funding}

The authors gratefully acknowledge the financial supports of the National Natural Science Foundation of China (21775023), Joint Funds for the innovation of science 


\section{A}

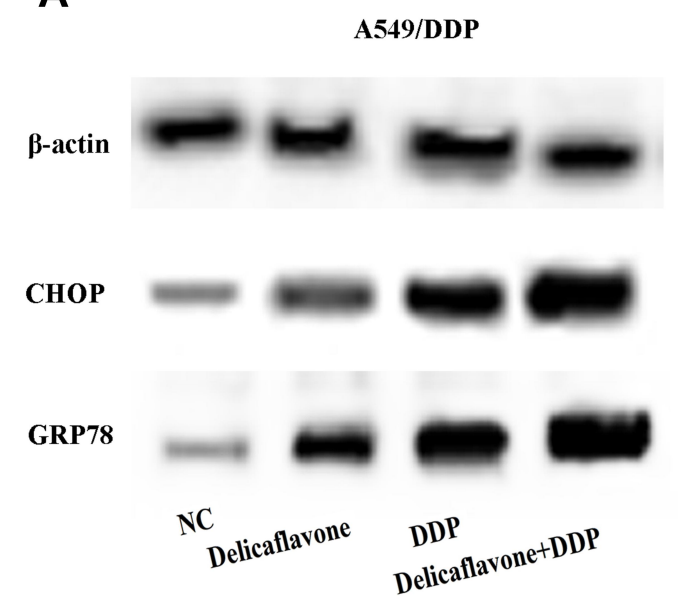

C

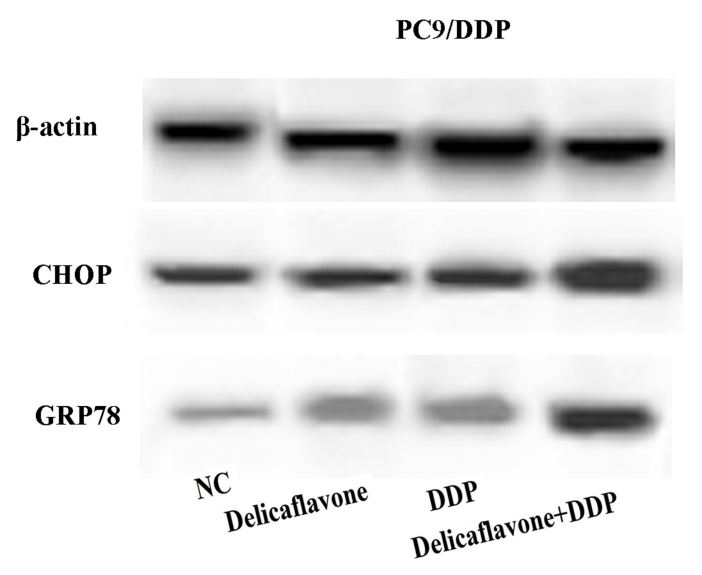

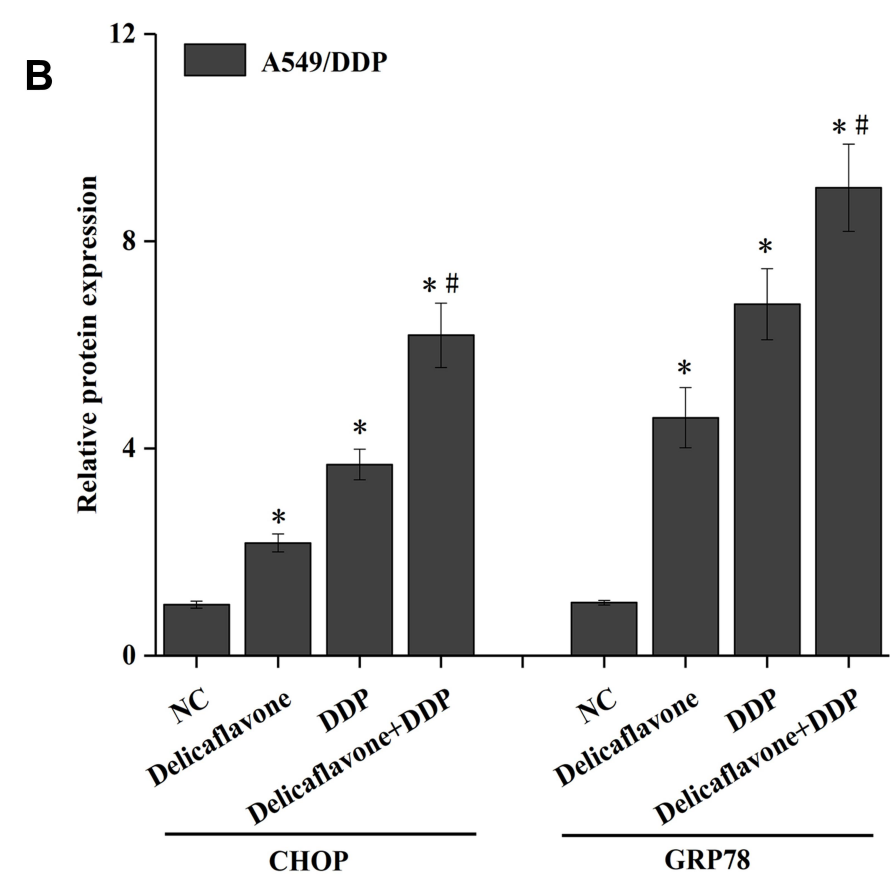

D

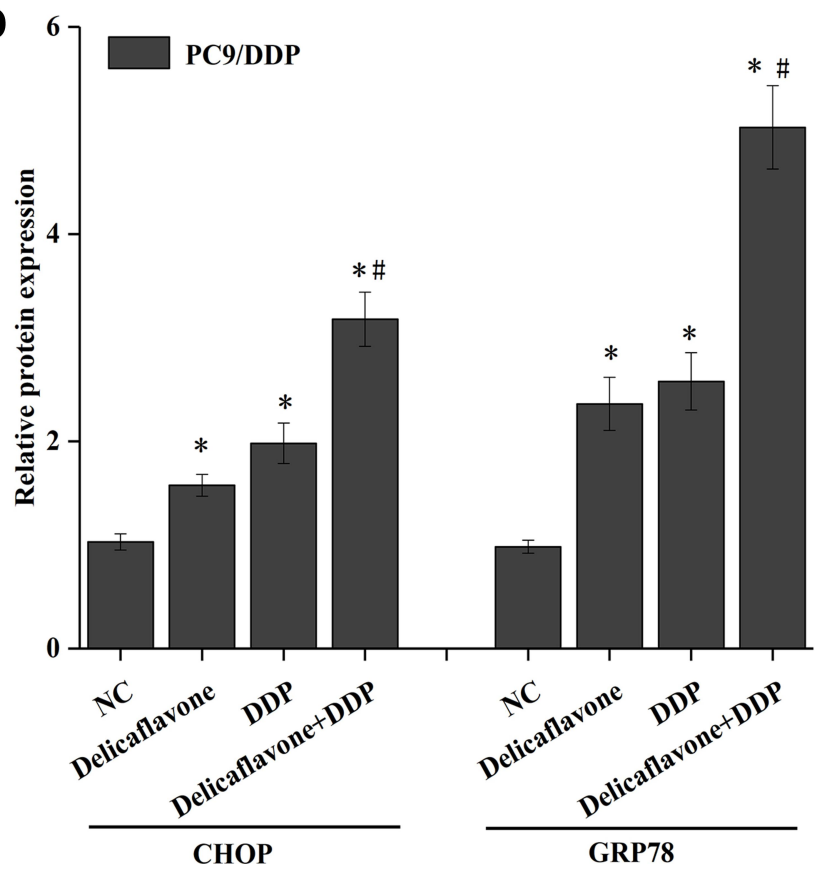

Figure 4 Delicaflavone united with DDP regulated lung cancer DDP resistant cells invasion, migration, apoptosis through ER stress signaling pathway. (A-D) The protein expression of ER stress-related protein CHOP and GRP78 were detected by Western blot. ${ }^{*} \mathrm{P}<0.05$ represents compared with control group. ${ }^{\#} \mathrm{P}<0.05$ represents compared with the Delicaflavone-alone group or DDP-alone group.

and Technology of Fujian province (2017Y9123, 2017 Y9124 and 2018Y9076), Health and Youth Research Project of Fujian province (2019-1-60), Funds for scientific research start-up of high-level talents of Fujian Medical University (XRCZX2018020), Guiding Project of Science and Technology Plan of Fujian Province (Grant number 2018Y0011), The Young and Middle-aged Talents
Training Project of Fujian Provincial Health Commission (Grant number 2018-ZQN-3), and Sailing Funds of Fujian Medical University (2018QH2013and 2018QH1016).

\section{Disclosure}

The authors report no conflicts of interest for this work. 


\section{References}

1. Bray F, Ferlay J, Soerjomataram I, et al. Global cancer statistics 2018: GLOBOCAN estimates of incidence and mortality worldwide for 36 cancers in 185 countries. CA Cancer J Clin. 2018;68 (6):394-424. doi:10.3322/caac. 21492

2. Santucci C, Carioli G, Bertuccio P, et al. Progress in cancer mortality, incidence, and survival: a global overview. Eur J Cancer Prev. 2020;29(5):367-381. doi:10.1097/CEJ.0000000000000594

3. Miranda-Filho A, Pineros M, Bray F. The descriptive epidemiology of lung cancer and tobacco control: a global overview 2018. Salud Publica Mex. 2019;61(3):219-229. doi:10.21149/10140

4. Chang JY, Verma V, Li M, et al. Proton beam radiotherapy and concurrent chemotherapy for unresectable stage III non-small cell lung cancer: final results of a Phase 2 study. JAMA Oncol. 2017;3 (8):e172032. doi:10.1001/jamaoncol.2017.2032

5. Peterson J, Niles C, Patel A, et al. Stereotactic body radiotherapy for large ( $>5 \mathrm{~cm}$ ) non-small-cell lung cancer. Clin Lung Cancer. 2017;18 (4):396-400. doi:10.1016/j.cllc.2016.11.020

6. Sun R, Wang R, Chang S, et al. Long non-coding RNA in drug resistance of non-small cell lung cancer: a mini review. Front Pharmacol. 2019;10:1457. doi:10.3389/fphar.2019.01457

7. Yao W, Lin Z, Wang G, et al. Delicaflavone induces apoptosis via mitochondrial pathway accompanying G2/M cycle arrest and inhibition of MAPK signaling cascades in cervical cancer HeLa cells. Phytomedicine. 2019;62:152973. doi:10.1016/j.phymed.2019.152973

8. Sui Y, Yao H, Li S, et al. Delicaflavone induces autophagic cell death in lung cancer via Akt/mTOR/p70S6K signaling pathway. J Mol Med (Berl). 2017;95(3):311-322. doi:10.1007/s00109-016-1487-z

9. Yao W, Lin Z, Shi P, et al. Delicaflavone induces ROS-mediated apoptosis and inhibits PI3K/AKT/mTOR and Ras/MEK/Erk signaling pathways in colorectal cancer cells. Biochem Pharmacol. 2019;171:113680. doi:10.1016/j.bcp.2019.113680

10. Molina JR, Yang P, Cassivi SD, et al. Non-small cell lung cancer: epidemiology, risk factors, treatment, and survivorship. Mayo Clin Proc. 2008;83(5):584-594. doi:10.1016/S0025-6196(11)60735-0

11. Li Z, Wang Y, Wu L, et al. Apurinic endonuclease 1 promotes the cisplatin resistance of lung cancer cells by inducing Parkinmediated mitophagy. Oncol Rep. 2019;42(6):2245-2254. doi:10.3892/ or.2019.7345

12. Jia J, Yang X, Zhao Q, et al. BNIP3 contributes to cisplatin-induced apoptosis in ovarian cancer cells. FEBS Open Biol. 2020;10 (8):1463-1473. doi:10.1002/2211-5463.12881

13. Guo H, Ren H, Li J, et al. TIPE2 suppressed cisplatin resistance by inducing autophagy via mTOR signalling pathway. Exp Mol Pathol. 2020;113:104367. doi:10.1016/j.yexmp.2020.104367
14. Xia A, Li H, Li R, et al. Co-treatment with BEZ235 enhances chemosensitivity of A549/DDP cells to cisplatin via inhibition of $\mathrm{PI} 3 \mathrm{~K} / \mathrm{Akt} / \mathrm{mTOR}$ signaling and downregulation of ERCC1 expression. Oncol Rep. 2018;40(4):2353-2362. doi:10.3892/ or. 2018.6583

15. Cernigliaro C, D'Anneo A, Carlisi D, et al. Ethanol-mediated stress promotes autophagic survival and aggressiveness of colon cancer cells via activation of Nrf2/HO-1 pathway. Cancers (Basel). 2019;11(4):505. doi:10.3390/cancers11040505

16. Vekaria PH, Kumar A, Subramaniam D, et al. Functional cooperativity of p97 and histone deacetylase 6 in mediating DNA repair in mantle cell lymphoma cells. Leukemia. 2019;33(7):1675-1686. doi:10.1038/s41375-018-0355-y

17. Teng X, Fan XF, Li Q, et al. XPC inhibition rescues cisplatin resistance via the Akt/mTOR signaling pathway in A549/DDP lung adenocarcinoma cells. Oncol Rep. 2019;41(3):1875-1882. doi:10.3892/or.2019.6959

18. Fang D, Xiong Z, Xu J, et al. Chemopreventive mechanisms of galangin against hepatocellular carcinoma: a review. Biomed Pharmacother. 2019;109:2054-2061. doi:10.1016/j.biopha.2018.09.154

19. Bahar E, Kim JY, Yoon H. Chemotherapy resistance explained through endoplasmic reticulum stress-dependent signaling. Cancers (Basel). 2019;11(3):338. doi:10.3390/cancers11030338

20. Alves-Fernandes DK, Oliveira EA, Faiao-Flores F, et al. ER stress promotes antitumor effects in BRAFi/MEKi resistant human melanoma induced by natural compound 4-nerolidylcathecol (4-NC). Pharmacol Res. 2019;141:63-72. doi:10.1016/j.phrs.2018.12.006

21. Yu L, Xie R, Tian T, et al. Suberoylanilide hydroxamic acid upregulates histone acetylation and activates endoplasmic reticulum stress to induce apoptosis in HepG2 liver cancer cells. Oncol Lett. 2019;18 (4):3537-3544. doi:10.3892/ol.2019.10705

22. Sisinni L, Pietrafesa M, Lepore S, et al. Endoplasmic reticulum stress and unfolded protein response in breast cancer: the balance between apoptosis and autophagy and its role in drug resistance. Int J Mol Sci. 2019;20(4):857. doi:10.3390/ijms20040857

23. Shi S, Tan P, Yan B, et al. ER stress and autophagy are involved in the apoptosis induced by cisplatin in human lung cancer cells. Oncol Rep. 2016;35(5):2606-2614. doi:10.3892/or.2016.4680

24. Hsu HH, Chen MC, Baskaran R, et al. Oxaliplatin resistance in colorectal cancer cells is mediated via activation of ABCG2 to alleviate ER stress induced apoptosis. J Cell Physiol. 2018;233 (7):5458-5467. doi:10.1002/jcp.26406

25. Szostakowska M, Trebinska-Stryjewska A, Grzybowska EA, et al. Resistance to endocrine therapy in breast cancer: molecular mechanisms and future goals. Breast Cancer Res Treat. 2019;173 (3):489-497. doi:10.1007/s10549-018-5023-4
OncoTargets and Therapy

\section{Publish your work in this journal}

OncoTargets and Therapy is an international, peer-reviewed, open access journal focusing on the pathological basis of all cancers, potential targets for therapy and treatment protocols employed to improve the management of cancer patients. The journal also focuses on the impact of management programs and new therapeutic agents and protocols on patient perspectives such as quality of life, adherence and satisfaction. The manuscript management system is completely online and includes a very quick and fair peer-review system, which is all easy to use. Visit http://www.dovepress.com/ testimonials.php to read real quotes from published authors. 